VOL. $61(2000)$ [27-32]

\title{
APPLICATIONS OF FOX'S DERIVATION IN DETERMINING THE GENERATORS OF A GROUP
}

\author{
WAN LIN
}

\begin{abstract}
We give a necessary and sufficient condition for a set of elements to be a generating set of a quotient group $F / N$, where $F$ is the free group of rank $n$ and $N$ is a normal subgroup of $F$. Birman's Inverse Function Theorem is a corollary of our criterion. As an application of this criterion, we give necessary and sufficient conditions for a set of elements of the Burnside group $B(n, p)$ of exponent $p$ and rank $n$ to be a generating set.
\end{abstract}

For a group $G$, denote by $\mathbb{Z} G$ its integral group ring. Elements of $\mathbb{Z} G$ are of the form $\sum_{g \in G} a_{g} g$, where $a_{g} \in \mathbb{Z}$ is equal to zero for all but a finite number of $g$. Addition and multiplication in $\mathbb{Z} G$ are defined by $\sum_{g \in G} a_{g} g+\sum_{g \in G} b_{g} g=\sum_{g \in G}\left(a_{g}+b_{g}\right) g$ and $\left(\sum_{g \in G} a_{g} g\right)\left(\sum_{g \in G} b_{g} g\right)=\sum_{g \in G}\left(\sum_{h \in G} a_{g h-1} b_{h}\right) g$. Let $\epsilon$ denote the augmentation homomorphism of $\mathbb{Z} G$ into itself, that is, $\left(\sum a_{g} g\right)^{e}=\sum a_{g}$, for any element $\sum a_{g} g$ in $\mathbb{Z} G$. The kernel $\Delta=\operatorname{Ker} \varepsilon$ is called the augmentation ideal of $\mathbb{Z} G$ [8]. It is easily verified that $\Delta=\mathbb{Z} G(G-1)$. A mapping $D$ of $\mathbb{Z} G$ into itself is called a (left) derivation if $D(u+v)=D u+D v$ and $D(u \cdot v)=D u \cdot v^{\varepsilon}+u \cdot D v$, for any $u, v$ in $\mathbb{Z} G$. Derivation in $\mathbb{Z} G$ has the following elementary properties:

(i) $D a=0$, for any $a \in \mathbb{Z}$;

(ii) $D\left(u_{1} \cdot u_{2} \cdots u_{k}\right)=\sum_{i=1}^{k} u_{1} \cdot u_{2} \cdots u_{i-1} \cdot D u_{i}$, for any elements $u_{1}^{i=1}, u_{2}, \ldots, u_{k} \in G, k \geq 1$;

(iii) $D\left(u^{-1}\right)=-u^{-1} \cdot D u$, for any element $u \in G$.

Let $F$ be the free group with basis $x_{1}, x_{2}, \ldots, x_{n}$. We let $\frac{\partial}{\partial x_{j}}, j=1,2, \ldots, n$, be the Fox free partial derivative with respect to $x_{j}$ in $\mathbb{Z F}[6,8]$, that is, $\frac{\partial x_{i}}{\partial x_{j}}=$ $\delta_{i j}$, where $\delta_{i j}$ is the Kroneker delta. If $y_{1}, y_{2}, \ldots, y_{n}$ is a set of elements of $F$, let

Received 23rd March, 1999

I would like to thank my supervisor Professor C.K. Gupta for suggesting this topic to me.

Copyright Clearance Centre, Inc. Serial-fee code: 0004-9727/00 \$A2.00+0.00. 
$\left\|\frac{\partial y_{i}}{\partial x_{j}}\right\|=\left\|\frac{\partial\left(y_{1}, y_{2}, \ldots, y_{n}\right)}{\partial\left(x_{1}, x_{2}, \ldots, x_{n}\right)}\right\|$ denote the Jacobian matrix. In 1973, Birman [4] proved that $y_{1}, y_{2}, \ldots, y_{n}$ generate $F$ if and only if $\left\|\frac{\partial y_{i}}{\partial x_{j}}\right\|$ has a right inverse.

In the current paper, we shall establish a necessary and sufficient condition for a set of elements $\overline{y_{1}}, \overline{y_{2}}, \ldots, \overline{y_{n}}$ to be a generating set for $G=F / N$, where $N$ is a normal subgroup of $F$. As an application of our criterion, we give necessary and sufficient conditions for a set of elements of the Burnside group $B(n, p)$ of exponent $p$ and rank $n$ to be a generating set.

THEOREM 1. Let $F$ be the free group with basis $x_{1}, x_{2}, \ldots, x_{n}$ and $N$ a normal subgroup of $F$. Suppose $y_{1}, y_{2}, \ldots, y_{n}$ is a set of elements of $F$. Then $\overline{y_{1}}, \overline{y_{2}}, \ldots$, $\overline{y_{n}}$ is a generating set for $F / N$ if and only if there exist an $n \times n$ matrix $A=\left(a_{i j}\right)$ over $\mathbb{Z} F$, elements $g_{1}, g_{2}, \ldots, g_{n}$ in $\mathbb{Z} F$ and $h_{1}, h_{2}, \ldots, h_{n}$ in $N$ such that

$$
\left(a_{i j}\right)\left\|\frac{\partial y_{i}}{\partial x_{j}}\right\|+\operatorname{diag}\left(g_{1}, g_{2}, \ldots, g_{n}\right)\left\|\frac{\partial h_{i}}{\partial x_{j}}\right\|=E
$$

where $E$ is the $n \times n$ identity matrix and $\operatorname{diag}\left(g_{1}, g_{2}, \ldots, g_{n}\right)$ is the $n \times n$ diagonal matrix with main diagonal entries $g_{1}, g_{2}, \ldots, g_{n}$.

Proof: If $\overline{y_{1}}, \overline{y_{2}}, \ldots, \overline{y_{n}}$ is a generating set of $F / N$, then $\overline{x_{i}}=X_{i}\left(\overline{y_{1}}, \overline{y_{2}}, \ldots, \overline{y_{n}}\right)$ is a word in $\overline{y_{1}}, \overline{y_{2}}, \ldots, \overline{y_{n}}$. Thus $x_{i}=W_{i}\left(y_{1}, y_{2}, \ldots, y_{n}\right) h_{i}$, where $h_{i} \in N$. In $\mathbb{Z} F$, we have that

$$
\delta_{i j}=\frac{\partial x_{i}}{\partial x_{j}}=\frac{\partial}{\partial x_{j}}\left\{W_{i}\left(y_{1}, y_{2}, \ldots, y_{n}\right) h_{i}\right\}=\frac{\partial W_{i}}{\partial x_{j}}+W_{i} \frac{\partial h_{i}}{\partial x_{j}}
$$

By property (ii), we may write $\frac{\partial W_{i}}{\partial x_{j}}$ as

$$
\frac{\partial W_{i}}{\partial x_{j}}=\sum_{k=1}^{n} a_{i k} \frac{\partial y_{k}}{\partial x_{j}},
$$

where $a_{i k}, 1 \leq k \leq n$, are elements in $\mathbb{Z} F$.

Let $A=\left(a_{i j}\right)$ and $g_{i}=W_{i}, i=1,2, \ldots, n$, then

$$
\left(a_{i j}\right)\left\|\frac{\partial y_{i}}{\partial x_{j}}\right\| \operatorname{diag}\left(g_{1}, g_{2}, \ldots, g_{n}\right)\left\|\frac{\partial h_{i}}{\partial x_{j}}\right\|=E
$$

Conversely, suppose that

$$
\left(a_{i j}\right)\left\|\frac{\partial y_{i}}{\partial x_{j}}\right\|+\operatorname{diag}\left(g_{1}, g_{2}, \ldots, g_{n}\right)\left\|\frac{\partial h_{i}}{\partial x_{j}}\right\|=E
$$


where $g_{1}, g_{2}, \ldots, g_{n} \in \mathbb{Z} F$ and $h_{1}, h_{2}, \ldots, h_{n} \in N$. Then

$$
\sum_{k=1}^{n} a_{i k} \frac{\partial y_{k}}{\partial x_{j}}+g_{i} \frac{\partial h_{i}}{\partial x_{j}}=\delta_{i j}
$$

Multiplying both sides of the above equation by $x_{j}-1$ and summing over $j$, we have

$$
\sum_{j=1}^{n} \sum_{k=1}^{n} a_{i k} \frac{\partial y_{k}}{\partial x_{j}}\left(x_{j}-1\right)+\sum_{j=1}^{n} g_{i} \frac{\partial h_{i}}{\partial x_{j}}\left(x_{j}-1\right)=\sum_{j=1}^{n} \delta_{i j}\left(x_{j}-1\right)
$$

By Fox's fundamental formula [6], we have that $\sum_{j=1}^{n} \frac{\partial y_{k}}{\partial x_{j}}\left(x_{j}-1\right)=y_{k}-1$ and $\sum_{j=1}^{n} \frac{\partial h_{i}}{\partial x_{j}}\left(x_{j}-1\right)=h_{i}-1$. Thus

$$
\sum_{k=1}^{n} a_{i k}\left(y_{k}-1\right)+g_{i}\left(h_{i}-1\right)=x_{i}-1
$$

Hence

$$
\sum_{k=1}^{n} a_{i k}\left(y_{k}-1\right)=x_{i}-1 \quad \bmod \mathbb{Z} F(N-1)
$$

It follows, by [5, Lemma 4.1], that $\overline{y_{1}}, \overline{y_{2}}, \ldots, \overline{y_{n}}$ is a generating set for $F / N$.

\section{REMARK.}

(i) The proof of the necessity actually gives an algorithm for finding the matrix $A$, elements $g_{1}, g_{2}, \ldots, g_{n}$ and $h_{1}, h_{2}, \ldots, h_{n}$. The elements $g_{1}, g_{2}, \ldots, g_{n}$ are actually in the free group $F$.

(ii) When $N=1$, Theorem 1 says that a set of elements $y_{1}, y_{2}, \ldots, y_{n}$ of $F$ is a basis for $F$ if and only if the Jacobian matrix $\left\|\frac{\partial y_{i}}{\partial x_{j}}\right\|$ has an inverse in $\mathbb{Z F}$. Thus Birman's Inverse Function Theorem [4] is a corollary of our Theorem 1.

As an application of Theorem 1, we shall prove the following result regarding the generating set of the Burnside group $B(n, p)$ of exponent $p$ and rank $n$. Let $p$ be a prime and $F^{p}$ the subgroup of $F$ generated by the $p$ th power of the elements of $F$. Then $B(n, p)=F / F^{p}$ is the Burnside group of exponent $p$ and rank $n$. We let $I_{p}$ denote the ideal of $\mathbb{Z} F$ generated by all elements of the form $1+w+w^{2}+\cdots+w^{p-1}$, $w \in F$. 
COROLlary 2. Let $y_{1}, y_{2}, \ldots, y_{n}$ be a set of elements of $F$. Then we have the following:

(i) If $\overline{y_{1}}, \overline{y_{2}}, \ldots, \overline{y_{n}}$ is a generating set of $B(n, p)$, then $\overline{\left\|\frac{\partial y_{i}}{\partial x_{j}}\right\|}$ is invertible over $\mathbb{Z} F / I_{p}$.

(ii) If $\left\|\frac{\partial y_{i}}{\partial x_{j}}\right\|$ has a left inverse over $\mathbb{Z} F / \mathbb{Z} F\left(F^{p}-1\right)$, then $\overline{y_{1}}, \overline{y_{2}}, \ldots, \overline{y_{n}}$ is a generating set of $B(n, p)$.

(iii) If $\left\|\frac{\partial y_{i}}{\partial x_{j}}\right\|$ has a left inverse over $\mathbb{Z} F / I_{p}$, then $\overline{y_{1}}, \overline{y_{2}}, \ldots, \overline{y_{n}}$ is a generating set of $F / N$, where $N$ is the normal subgroup of $F$ corresponding to $I_{p}$, that is, $I_{p}=\mathbb{Z} F(N-1)$.

Proof: (i) If $\overline{y_{1}}, \overline{y_{2}}, \ldots, \overline{y_{n}}$ is a generating set of $B(n, p)$, then, by Theorem 1 , there exist an $n \times n$ matrix $A=\left(a_{i j}\right)$, elements $g_{1}, g_{2}, \ldots, g_{n}$ in $F$ and elements $h_{1}, h_{2}, \ldots, h_{n}$ in $F^{p}$ such that

$$
\left(a_{i j}\right)\left\|\frac{\partial y_{i}}{\partial x_{j}}\right\|+\operatorname{diag}\left(g_{1}, g_{2}, \ldots, g_{n}\right)\left\|\frac{\partial h_{i}}{\partial x_{j}}\right\|=E .
$$

Since $h_{i} \in F^{p}, 1 \leq i \leq n$, we have that $\frac{\partial h_{i}}{\partial x_{j}} \in I_{p}$ for all $i$ and $j$. Thus

$$
\left(a_{i j}\right)\left\|\frac{\partial y_{i}}{\partial x_{j}}\right\|=E
$$

over $\mathbb{Z} F / I_{p}$. Hence $\left(a_{i j}\right)$ is a left inverse of $\overline{\left\|\frac{\partial y_{i}}{\partial x_{j}}\right\|}$ over $\mathbb{Z} F / I_{p}$. By a theorem of Montgomery $[9],\left(a_{i j}\right)$ is also a right inverse of $\left\|\frac{\partial y_{i}}{\partial x_{j}}\right\|$. Therefore $\left(a_{i j}\right)$ is the inverse of $\overline{\left\|\frac{\partial y_{i}}{\partial x_{j}}\right\|}$.

(ii) Suppose that

$$
\left(a_{i j}\right)\left\|\frac{\partial y_{i}}{\partial x_{j}}\right\|=E
$$

over $\mathbb{Z} F / \mathbb{Z} F\left(F^{p}-1\right)$. Then we can find $u_{i j}$ in $\mathbb{Z} F\left(F^{p}-1\right)$ such that

$$
\left(a_{i j}\right)\left\|\frac{\partial y_{i}}{\partial x_{j}}\right\|+\left(u_{i j}\right)=E
$$

over $\mathbb{Z} F$. Thus, for $i=1,2, \ldots, n$ and $j=1,2, \ldots, n$,

$$
\sum_{k=1}^{n} a_{i k} \frac{\partial y_{k}}{\partial x_{j}}+u_{i j}=\delta_{i j}
$$


Multiplying both sides of this equation by $x_{j}-1$ and summing over $j$, we have

$$
\sum_{j=1}^{n} \sum_{k=1}^{n} a_{i k} \frac{\partial y_{k}}{\partial x_{j}}\left(x_{j}-1\right)+\sum_{j=1}^{n} u_{i j}\left(x_{j}-1\right)=\sum_{j=1}^{n} \delta_{i j}\left(x_{j}-1\right) .
$$

By Fox's fundamental formula, we have that

$$
\sum_{k=1}^{n} a_{i k}\left(y_{k}-1\right)+\sum_{j=1}^{n} u_{i j}\left(x_{j}-1\right)=x_{i}-1 .
$$

Thus

$$
\sum_{k=1}^{n} a_{i k}\left(y_{k}-1\right)=x_{i}-1 \bmod \mathbb{Z} F\left(F^{p}-1\right) .
$$

Therefore, by $[5$, Lemma 4.1$], \overline{y_{1}}, \overline{y_{2}}, \ldots, \overline{y_{n}}$ is a generating set for $B(n, p)$.

(iii) If

$$
\left(a_{i j}\right)\left\|\frac{\partial y_{i}}{\partial x_{j}}\right\|=E
$$

over $\mathbb{Z} F / I_{p}$, then we can find $u_{i j}$ in $I_{p}$ such that

$$
\left(a_{i j}\right)\left\|\frac{\partial y_{i}}{\partial x_{j}}\right\|+\left(u_{i j}\right)=E
$$

over $\mathbb{Z} F$. Thus, for $i=1,2, \ldots, n$ and $j=1,2, \ldots, n$,

$$
\sum_{k=1}^{n} a_{i k} \frac{\partial y_{k}}{\partial x_{j}}+u_{i j}=\delta_{i j}
$$

Multiplying both sides of this equation by $x_{j}-1$ and summing over $j$, we have

$$
\sum_{j=1}^{n} \sum_{k=1}^{n} a_{i k} \frac{\partial y_{k}}{\partial x_{j}}\left(x_{j}-1\right)+\sum_{j=1}^{n} u_{i j}\left(x_{j}-1\right)=\sum_{j=1}^{n} \delta_{i j}\left(x_{j}-1\right) .
$$

By Fox's fundamental formula, we have

$$
\sum_{k=1}^{n} a_{i k}\left(y_{k}-1\right)+\sum_{j=1}^{n} u_{i j}\left(x_{j}-1\right)=x_{i}-1
$$

Thus

$$
\sum_{k=1}^{n} a_{i k}\left(y_{k}-1\right)=x_{i}-1 \bmod I_{p}
$$

Let $N$ be the normal subgroup of $F$ such that $I_{p}=\mathbf{Z} F(N-1)$, then $N \supseteq F^{p}$. Thus, by [5, Lemma 4.1], $\overline{y_{1}}, \overline{y_{2}}, \ldots, \overline{y_{n}}$ is a generating set for $F / N$. 


\section{REFERENCES}

[1] S. Bachmuth, 'Automorphisms of free metabelian groups', Trans. Amer. Math. Soc. 118 (1965), 93-104.

[2] S. Bachmuth, 'Automorphisms of a class of metabelian groups', Trans. Amer. Math. Soc. 127 (1967), 284-293.

[3] S. Bachmuth and H.Y. Mochizuki, 'Automorphisms of a class of metabelian groups II', Trans. Amer. Math. Soc. 127 (1967), 294-301.

[4] J.S. Birman, 'An inverse function theorem for free groups', Proc. Amer. Math. Soc. $\mathbf{4 1}$ (1973), 634-638.

[5] D.E. Cohen, Groups of cohomological dimension one, Lecture Notes in Math. 245 (Springer-Verlag, Berlin, Heidelberg, New York, 1972).

[6] R.H. Fox, 'Free differential calculus I, Derivation in the free group ring', Ann. of Math. $57(1953), 547-560$.

[7] R.H. Fox, 'Free differential calculus II, The isomorphism problem of groups', Ann. of Math. 59 (1954), 196-210.

[8] N.D. Gupta, Free group rings, Contemporary Mathematics 66 (Amer. Math. Soc., Providence, RI, 1987).

[9] M.S. Montgomery, 'Left and right inverses in group algebras', Bull. Amer. Math. Soc. 75 (1969), 539-540.

\footnotetext{
Department of Mathematics

University of Manitoba

Winnipeg

Manitoba R3T 2N2

Canada

e-mail: umlin010@cc.umanitoba.ca
} 